\title{
Future Therapeutic Concepts
}

\section{U. Deniz Dincer}

Ufuk University School of Medicine. Ankara, Turkey

The natural history of metabolic syndrome has been wildly described which manifested with cardio metabolic risk such as hypertension and dyslipidemia together with diabetes mellitus or not. Unfortunately, increasing burden of childhood obesity especially enhanced body mass index specifically centrally distributed adiposity trigger prevalence of metabolic syndrome in western countries. To help clinicians to cope with metabolic syndrome associated complications based on latest guidelines still require increase in early diagnosis of the disease. Main treatment strategies cover strict body weight control, blood pressure management, blood lipids normalization with good glycemic aspects secondary to increase life style quality with right diet modification. The link between metabolic syndrome and cardiovascular risk is clear as possible however, to be overweight is accept independent risk factor but insulin resistance and dyslipidemia appear integral components of the disease. The primary end points of cardio vascular metabolic risk profile display coronary and peripheral arterial disease, myocardial infarction, congestive heart failure, arrhythmias and stroke. Reduced $\mathrm{HbA1c}$, low density lipoprotein cholesterol (LDL-C), triglycerides and increased high density lipoproteins (HDL-C) are very well known benefits of decrease future cardio metabolic risk. All these algorithms modify new treatment concepts to explore best future pharmaceutical contents against cardiovascular complication.

New therapeutic strategies to protect cardiac and hemodynamic functions in metabolic syndrome focus on ultimately normalize constant sympathetic system activity and hyperdynamic circulatory state. $\beta_{1}$ adrenoceptor (AR) antagonists, angiotensin receptor blockers (AT1), angiotensin-converting enzyme (ACE) inhibitors, renin antagonists able to help block hazardous sides of compensatory mechanisms. Additional to angiotensin antagonists, mineralacorticoid (MR)-receptor blockers, xanthine oxidase inhibitors (XO-I) and HMG-coA-reductase inhibitors (statin) also display various beneficial cellular benefits against elevated levels of reactive oxygen species (ROS) and impaired vascular reactivity. JTV519 (K201) is a newly developed 1,4-benzothiazepine drug with antiarrhythmic and cardioprotective properties that has been shown to increase the affinity of calstabin (FKBP12.6) for cardiac ryanodine receptor $\left(\mathrm{RyR}_{2}\right)$. It blocks L-type $\mathrm{Ca}^{++}$current like diltiazem which is relatively non selective blocker of cation currents. K201 (JTV-519) increases binding affinity of FKBP12.6 to $\mathrm{RyR}_{2}$, which stabilizes the closes state of Ry $\mathrm{R}_{2}$ channels and prevents $\mathrm{Ca}^{++}$leak. It protects from ventricular arrhythmias, contractile dysfunction and reduce $\mathrm{Ca}^{++}$overload. These beneficial combinations dramatically ameliorate the progression of heart against to myocardial damage resulting from $\mathrm{Ca}^{++}$overload. The main beneficial effect of the JTV519 (K201) is appear to reduce the abnormal $\mathrm{Ca}^{++}$leak throughthe increase binding affinity of FKBP12.6 to Ry $\mathrm{R}_{2}$. Enhancing binding affinity of FKBP12.6 to $\mathrm{RyR}_{2}$ with JTV519 may be a first future therapeutic strategy to treat cardiac disturbances in metabolic syndrome especially, appear to improve exercise-induced arrhythmias.

Newer therapeutic agents also are available in metabolic syndrome if the disease together with diabetes. The glucagon like peptide (GLP-I) analogue such as exenatide included randomized controlled clinical trials demonstrate that it is effective in improving glycemic control together with beneficial weight loss. The other newer agents "dipeptidyl peptidase-4 (DPP-4) inhibitor" gliptins (such as sitagliptin and vildagliptin) included clinical trials demonstrate they are effective in management of glycemic control even that they represent lower hypoglycemia episodes and less weight gain problem. Some knowledge already exists regarding they reduce $\mathrm{HbA}{ }_{c}$ level couple of percent and decrease hyperglycemic/hypoglycemic frequent especially nocturnal ones.

A paradigm is still on regarding which therapy should initiate first and when. For that reason, key market players never give up and still willing to deal to develop new molecules. The challenging point in this arena is emerging therapeutic agents seems to be not very effective because this is complex, multifactorial and multifaceted syndrome and together with cardiovascular complications.
Corresponding author: U. DenizDincer MD, PhD, Ufuk University School of Medicine. Ankara, Turkey, E-mail: uddincer@ufuk.edu.tr

Received December 08, 2011; Accepted December 10, 2012; Published December 13, 2011

Citation: Dincer UD (2011) Future Therapeutic Concepts. J Metabolic Synd 1:e102. doi:10.4172/2167-0943.1000e102

Copyright: ( 2012 Dincer UD. This is an open-access article distributed under the terms of the Creative Commons Attribution License, which permits unrestricted use, distribution, and reproduction in any medium, provided the original author and source are credited. 